\title{
Anti-neutrophil cytoplasmic antibody-negative microscopic polyangiitis: A case report and literature review
}

\author{
RAN WANG $^{1}$, XIAO-CHUN YANG ${ }^{1}$, SI-JING ZHOU ${ }^{2}$ and GENG-YUN SUN ${ }^{1}$ \\ ${ }^{1}$ Department of Respiratory Medicine, The First Affiliated Hospital of Anhui Medical University; \\ ${ }^{2}$ Hefei Prevention and Treatment Center for Occupational Diseases, Hefei, Anhui 230022, P.R. China
}

Received October 31, 2014; Accepted May 8, 2015

DOI: $10.3892 /$ etm.2015.2523

\begin{abstract}
The aim of this study was to investigate the clinical characteristics, diagnosis and treatment of anti-neutrophil cytoplasmic antibody (ANCA)-negative microscopic polyangiitis (MPA). We described the case of a patient with ANCA-negative MPA and conducted analyses and a review of the relevant literature. Based on the collected data, the epidemiology, diagnosis and treatment of ANCA-negative MPA were discussed. The patient, a 69-year-old male, was initially diagnosed with pneumonia and interstitial lung disease (ILD) based on his clinical symptoms. The patient was ANCA-negative. The follow-up and consultations with the relevant departments after the ANCA testing led to a diagnosis of MPA being considered. The administration of glucocorticoids and immunosuppressant drugs was found to improve the symptoms of the patient. The clinical symptoms of MPA are unspecific. The majority of MPA cases are ANCA-positive, but misdiagnosis should be considered as a possibility in ANCA-negative cases. When patients are suspected to have MPA, therefore, ANCA tests should be immediately performed. Test results should be analyzed for the early diagnosis of MPA in order to enable the provision of immediate treatment, improve patient prognosis and reduce mortality rate.
\end{abstract}

\section{Introduction}

Microscopic polyangiitis (MPA) is a systemic vasculitis involving small- and medium-sized vessels and is associated with a focal and segmental necrotizing glomerulonephritis. MPA may be characterized as a pulmonary-renal syndrome with rapidly progressive glomerulonephritis and alveolar hemorrhage; however, the manifestations of the disease vary depending on the organ systems involved (1). The incidence

Correspondence to: Dr Geng-Yun Sun, Department of Respiratory Medicine, The First Affiliated Hospital of Anhui Medical University, 218 Jixi Road, Hefei, Anhui 230022, P.R. China

E-mail: sungengyun@hotmail.com

Key words: anti-neutrophil cytoplasmic antibody, microscopic polyangiitis, glucocorticoids and diagnosis rates of vasculitis have recently been rising constantly. The detection rate of anti-neutrophil cytoplasmic antibodies (ANCAs) in cases of vasculitis can reach as high as $90 \%$ (2). The detection of ANCA in the serum has therefore become an important basis for the diagnosis of vasculitis (3). ANCA-negative vasculitis is relatively rare and is thus prone to misdiagnosis. In this study, we describe a case of microscopic polyangiitis (MPA) and review the relevant literature with the aim of enhancing the clinical understanding of ANCA-negative vasculitis and improving the diagnosis and treatment of the condition.

\section{Case report}

The patient was a 69-year-old male who was admitted to the Department of Respiratory Medicine of the First Affiliated Hospital of Anhui Medical University (Hefei, China) due to 'fever with dyspnea for $>40$ days' on March 7, 2014. The highest measured body temperature of the patient reached up to $40^{\circ} \mathrm{C}$, usually in the afternoon and at night. He was admitted to another hospital and given an initial diagnosis of upper respiratory tract infection, which then became pneumonia. The patient was treated with multiple broad-spectrum antibiotics, to fight the infection, and hormones; however, the health of the patient did not improve, and the dyspnea eventually emerged. The outpatient service sent the patient to our department due to the supposed pneumonia.

Upon physical examination, mild cyanosis was observed in the patient's lips. The breath sounds from both lungs were coarse. Velcro-like rales could be heard from the base of both lungs. The heart rate was 108 beats/min, and the rhythm was regular. No edema was observed in either lower limb. Laboratory examination was conducted subsequent to admission. The results from the routine blood examination showed a white blood cell count of $8.49 \times 10^{9}$ cells $/ 1$, a neutrophil percentage of $79.44 \%$, a red blood cell count of $3.84 \times 10^{12}$ cells/ 1 and a platelet count of $3.30 \times 10^{11}$ cells/l. Routine examination yielded the following results: Occult blood in urine $(++)$; qualitative urine protein (+); erythrocyte sedimentation rate (ESR), $55 \mathrm{~mm} / \mathrm{h}$; C-reactive protein, $167.2 \mathrm{mg} / \mathrm{l}$; ANCA, 13 anti-nuclear antibodies, negative; normal renal function and tumor markers; and no pathogenic bacterial growth in the sputum culture. The ' $G$ ' and galactomannan tests were negative. The test for 13 antinuclear antibodies was also negative. 
Based on the test results, a diagnosis of interstitial lung disease (ILD) and pulmonary infection was reached. Informed consent was obtained from the patient prior to treatment. The patient was treated with anti-infection and phlegm elimination drugs with nutritional support; however, the patient's symptoms did not improve. Chest computed tomography (CT) in another hospital showed interstitial changes in both lungs (Fig. 1). The chest CT was reviewed on March 9. The result showed that the transparency in both lungs was reduced, and patchy, high-density strip shadows were diffusely distributed and interwoven into a grid shape (Fig. 2). Although the test result for ANCA was negative, a diagnosis of ANCA-associated vasculitis was considered. The possibility of MPA was great, but tuberculosis, fungal infection and lung cancer were excluded.

The patient was treated with methylprednisolone $(40 \mathrm{mg}$ every $12 \mathrm{~h}$ ) and continuous anti-infection drugs; however, the patient's temperature control was poor and the dyspnea was not significantly relieved. In addition, the perinuclear type was reviewed, showing positive results for ANCA (1:80). Positive results for anti-myeloperoxidase (MPO) antibody were also obtained. The patient agreed to the addition of cyclophosphamide for treatment. Through this treatment, the body temperature of the patient dropped to the normal range, and the dyspnea was relieved. The chest CT on March 30 showed that the diffuse, patchy, high-density strip shadows in both lungs were significantly absorbed (Fig. 3). The doses of administered steroid and cyclophosphamide were gradually reduced. In the course of treatment, the detected perinuclear types of ANCA were 1:40 and 1:10, whereas the cytoplasmic ANCA tests were negative. The routine urine test results, renal function and ESR were repeatedly reviewed, and all results showed that the patient's illness was gradually improving. The changes in the condition of the patient supported the diagnosis of MPA. The patient was again examined in May 2014, and the tests results for ANCA and ESR were normal. The patient's general condition was good.

\section{Discussion}

Vasculitis refers to a series of heterogeneous diseases occurring in the vascular wall and its surroundings, which may present with or without necrosis and can result in different degrees of stenosis or damage to the vessels and ischemic damage to the innervated tissues or organs. The majority of vasculitis cases are primary, often referred to as systemic vasculitis (4). Primary systemic vasculitis affecting the lungs includes pulmonary capillaritis, ANCA-associated vasculitis, Wegener's granulomatosis, Churg-Strauss syndrome and MPA. MPA is a systemic necrotizing vasculitis that affects small vessels. This condition can invade arterioles, microarteries, capillaries and venules of the kidneys, skin, lungs and other organs and is often detected as necrotizing glomerulonephritis and pulmonary capillaritis. The disease is known as MPA due to it mainly affecting small vessels, including veins (1). The incidence rate of MPA is (1-3)/100,000 in countries outside of China (5); in China, the incidence is unclear. With the recent increased frequency of ANCA examination, however, the number of diagnosed cases has shown a considerable increase. MPA predominantly occurs in males, mostly $50-60$ years old.

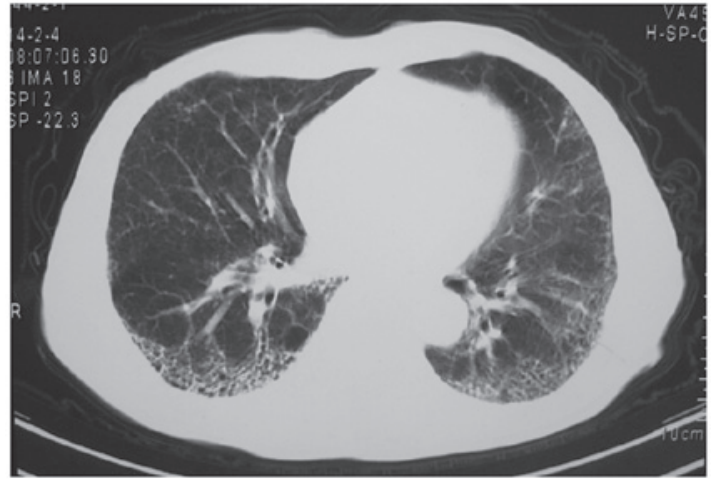

Figure 1. Interstitial changes in the inferior lungs, as shown by chest computed tomography in another hospital.

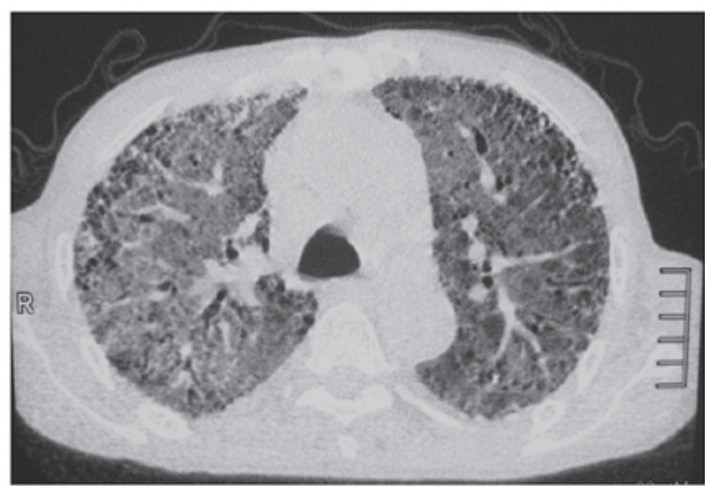

Figure 2. Transparency in the lungs was reduced, and patchy, high-density strip shadows were diffusely distributed and interwoven into a grid shape.

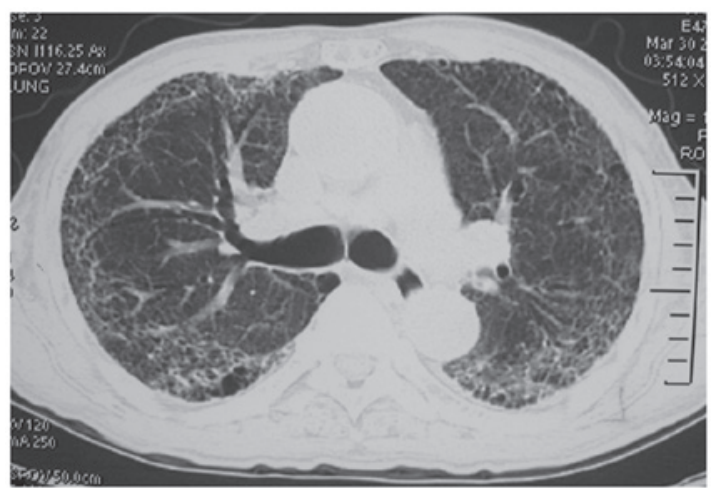

Figure 3. Diffuse lesions in the lungs were more significantly absorbed than previously.

The course of MPA varies; sometimes, the onset is acute, with rapidly progressive glomerulonephritis and pulmonary hemorrhage (6); sometimes, the onset is insidious with intermittent purpura, mild renal damage and intermittent hemoptysis. The disease often involves the kidney, which can cause necrotizing crescentic glomerulonephritis. Furthermore, MPA can involve multiple tissues and organs, including the lungs, skin, joints and digestive tract (6).

According to the literature, lungs infected with ANCA-associated vasculitis with different etiologies exhibit different characteristics. MPA mainly manifests as pulmonary 
infiltration, diffuse ILD and diffuse alveolar hemorrhage. The patient in the present report was admitted due to 'fever and dyspnea for $>40$ days'. The patient's renal function was normal, without cutaneous change, and his chest $\mathrm{CT}$ in another hospital showed interstitial lesions in both lungs. Following the admission of the patient, he tested negative for ANCA; therefore, the early diagnosis was unclear. With the progression of the disease, it was observed that i) the pulmonary lesions progressed rapidly; ii) the routine urine test results of the patient showed occult blood and urine protein, which could not be interpreted as being associated with pulmonary infection; and iii) the symptoms did not improve significantly following anti-infection treatment. Chest CT showed that the lesions in both lungs progressed more quickly than previously. During hospitalization, follow-up tests for ANCA and consultations with the appropriate departments were conducted. The final diagnosis was then made.

MPA lacks specific clinical manifestations and is thus easily misdiagnosed if the pulmonary symptoms are taken as the main indicators when other clinical symptoms are unspecific. Pulmonary interstitial lesions can be easily diagnosed but can be typically mistaken for connective tissue disease or idiopathic pulmonary fibrosis rather than MPA, thus resulting in the high incidence rate of early misdiagnosis and poor prognosis.

It has previously been reported that pulmonary injury in patients with MPA occurs secondary to kidney damage (7). Chen et al (3) found that, among patients with ANCA-associated vasculitis, patients aged $>65$ years exhibited a significantly higher incidence of pulmonary infection than patients aged $<65$ years. The basic pulmonary pathology was pulmonary capillary inflammation or necrotizing granulomatous vasculitis. A clinical diagnosis of MPA could be considered in the following settings: i) Patients showing such symptoms as fever, cough, expectoration, hemoptysis and dyspnea; interstitial changes in the lungs shown by chest imaging; detection of fungi and tuberculosis by sputum culture and smear; exclusion of infectious factors; and inefficacy of anti-infection and anti-tuberculosis treatments; ii) involvement of multiple organs from different systems, such as kidneys, lungs, skin, joints and nerves; and iii) notably increased ESR. If the patient is suspected to suffer from MPA, then ANCA examination should be performed to confirm the diagnosis (8).

The diagnosis of MPA lacks a uniform standard. If multiple system damage, pulmonary and renal infection or palpable purpura emerge, the diagnosis of MPA should be considered, particularly for patients testing positive for perinuclear ANCA. Renal, cutaneous or other visceral biopsies may be of use in the diagnosis of MPA; however, infective endocarditis has been excluded for patients. Studies have reported that $>80 \%$ of patients with MPA are ANCA-positive (8-11); therefore, a diagnosis of MPA cannot be excluded for ANCA-negative patients. Renal or other biopsies should be suggested (12). When the patient tests negative for ANCA, the result could be a false negative. A variety of target antigens are aimed at treating ANCA. These antigens include MPO and proteinase 3; however, ANCA has other subtypes, which cannot be completely distinguished from serum ANCA-negative vasculitis (2).

With the progression of the disease in the present case, the patient tested positive for ANCA. Previous studies have revealed that the ANCA titer is usually associated with the activity of the vasculitis and can reflect the curative effect to a certain extent $(13,14)$. The follow-up examination of the patient showed that, following treatment with glucocorticoids and cyclophosphamide, the ANCA titer gradually decreased, which was consistent with the literature.

Certain scholars believe that a definite diagnosis for patients with MPA, particularly those who are ANCA-negative, depends on pathological examination (8). In the present case, respiratory failure was apparent in the patient during hospitalization, which made renal and pulmonary biopsies unsuitable. Furthermore, the patient had no cutaneous lesions, and performing other relatively safe biopsies was infeasible. The patient fell ill with fever and expiratory dyspnea. The initial diagnosis was lung infection, and anti-infection treatment was ineffective; however, a definitive diagnosis was ultimately made on the basis of medical history, routine urine and ANCA tests and chest CT. The patient was treated with glucocorticoids and immunosuppressive therapy, and the symptoms significantly eased. The ANCA titer and ESR decreased, and the chest CT improved. The results supported the diagnosis of MPA.

In summary, MPA is a disease involving multiple systems. Its clinical manifestation is complicated and changeable, and the illness rapidly develops. In the present case, the initial symptoms of the patient pointed to a diagnosis of pulmonary infection, but pathological examination could not be performed, which lent difficulty to the clinical diagnosis. By integrating the clinical symptoms, ANCA detection results and chest $\mathrm{CT}$, a clinical diagnosis was reached. Combined with a review of the literature, we conclude that tests for ANCA should be promptly improved and that changes in the results should be monitored for patients with suspected MPA. Efforts should be made to improve the early discovery and diagnosis rates and to achieve timely medication for MPA to improve the patient prognosis while reducing mortality.

\section{Acknowledgements}

This study was supported by a grant from the State Key Clinical Specialty Construction Project (no. 2012AH001), the fund for the Academic Backbone of the Excellent Young and Middle-age People of Anhui Medical University (no. 2013010) and the fund from the First Affiliated Hospital of Anhui Medical University for Reserve Talents (no. 2014005).

\section{References}

1. Smyth L, Gaskin G and Pusey CD: Microscopic polyangiitis. Semin Respir Crit Care Med 25: 523-533, 2004.

2. Collins CE and Quismorio FP Jr: Pulmonary involvement in microscopic polyangiitis. Current Opin Pulm Med 11: 447-451, 2005.

3. Chen M, Yu F, Zhang Y and Zhao MH: Antineutrophil cytoplasmic autoantibody-associated vasculitis in older patients. Medicine (Baltimore) 87: 203-209, 2008.

4. Scott DG and Watts RA: Epidemiology and clinical features of systemic vasculitis. Clin Exp Nephrol 17: 607-610, 2013.

5. Tzelepis GE, Kokosi M, Tzioufas A, Toya SP, Boki KA, Zormpala A and Moutsopoulos HM: Prevalence and outcome of pulmonary fibrosis in microscopic polyangiitis. Eur Respir J 36: 116-121, 2010.

6. Villiger PM and Guillevin L: Microscopic polyangiitis: Clinical presentation. Autoimmun Rev 9: 812-819, 2010.

7. Eschun GM, Mink SN and Sharma S: Pulmonary interstitial fibrosis as a presenting manifestation in perinuclear antineutrophilic cytoplasmic antibody microscopic polyangiitis. Chest 123 : 297-301, 2003. 
8. Kallenberg CG: The diagnosis and classification of microscopic polyangiitis. J Autoimmunity 48-49: 90-93, 2014.

9. Wilke L, Prince-Fiocco M and Fiocco GP: Microscopic polyangiitis: A large single-center series. J Clin Rheumatol 20: 179-182, 2014.

10. Langford CA: Cyclophosphamide as induction therapy for Wegener's granulomatosis and microscopic polyangiitis. Clin Exp Immunol 164 (Suppl 1): 31-34, 2011.

11. Gibelin A, Maldini C and Mahr A: Epidemiology and etiology of wegener granulomatosis, microscopic polyangiitis, churg-strauss syndrome and goodpasture syndrome: Vasculitides with frequent lung involvement. Semin Respir Crit Care Med 32: 264-273, 2011.
12. Chung SA and Seo P: Microscopic polyangiitis. Rheum Dis Clin North Am 36: 545-558, 2010.

13. Kuboshima S, Tsuruoka K, Shirai S, Sasaki H, Sakurada T, Miura H, Okabayashi J, Konno Y, Shima Y, Yasuda T, et al: An autopsy case of microscopic polyangiitis complicated with pulmonary aspergilloma and cytomegalovirus pneumonia. Nihon Jinzo Gakkai Shi 49: 125-129, 2007 (In Japanese).

14. Zhao MH and Lockwood CM: ANCA defines the clinical disease manifestations of vasculitis. Sarcoidosis Vasc Diffuse Lung Dis 13: 221-226, 1996. 